Int J Theor Phys manuscript No.

(will be inserted by the editor)

F. Rahaman - S. Chakraborty - Saibal

Ray · A. A. Usmani · S. Islam

\title{
The higher dimensional gravastars
}

Received: date / Accepted: date

\begin{abstract}
A new model of gravastar is obtained in $D$-dimensional Einstein gravity. This class of solutions includes the gravastar as an alternative to $D$ dimensional versions of the Schwarzschild-Tangherlini black hole. The configuration of this new gravastar consists of three different regions with different equations of state: [I] Interior: $0 \leq r<r_{1}, \rho=-p$; [II] Shell: $r_{1} \leq r<r_{2}$, $\rho=p$; [III Exterior: $r_{2}<r, \rho=p=0$. The outer region of this gravastar corresponds to a higher dimensional Schwarzschild-Tangherlini black hole.
\end{abstract}

Keywords General Relativity; Gravastar; Higher Dimension

\section{Introduction}

Our recent model of a charge free gravastar in $(2+1)$-dimensional anti-de Sitter spacetime 1 and its subsequent generalization to charged gravastars with electrovacuum exterior [2] express perfectly the profound success of our

\section{F. Rahaman}

Department of Mathematics, Jadavpur University, Kolkata 700032, India E-mail: rahaman@iucaa.ernet.in

S. Chakraborty

Department of Mathematics, Jadavpur University, Kolkata 700032, India E-mail: schakraborty@math.jdvu.ac.in

Saibal Ray

Department of Physics, Government College of Engineering \& Ceramic Technology, Kolkata 700010, West Bengal, India E-mail: saibal@iucaa.ernet.in

A.A. Usmani

Department of Physics, Aligarh Muslim University, Aligarh 202002, Uttar Pradesh, India E-mail: anisul@iucaa.ernet.in

S. Islam Department of Mathematics, Jadavpur University, Kolkata 700032, West Bengal, India E-mail: sofiqul001@yahoo.co.in 
efforts in constructing a non-singular gravastar as an alternative to $(2+$ 1) black holes. These efforts were inspired by an earlier attempt by us 3 , wherein we had constructed a compact astrophysical charged object, a (3+ 1)-dimensional charged gravastar, as an alternative to charged black holes. However, present solution for the proposed astrophysical object of higher dimensional gravastars is found to be singular at its origin, which is a point of worry. Thus, there is a pertinent need to understand the subject from the very basics of cleaner $(2+1)$-dimensional gravity and develop the subject of these gravitational vacuum stars starting from $(2+1)$-dimensions to higher dimensions.

In connection to de Sitter spacetime and black holes a series of works are available in the literature $4,5,6,7,8,9,10,11$. These investigations are interesting in the sense that the authors have analyzed the globally regular solution of the Einstein equations describing a black hole whose singularity is replaced by the de Sitter core.

However, in our present study we extend the proposition of charge free gravastars of Mazur and Mottola [12,13] to a charged compact object. While doing so, we invoke the very idea of electromagnetic mass (EMM) which suggests that interior de Sitter vacuum of a charged gravastar generates gravitational mass 14, 15, 16, 17. This provides a stable configuration by balancing the repulsive pressure arising from charge with its alternative gravity to avert a singularity.

It is a common trend to believe that the 4-dimensional present spacetime structure is the self-compactified form of manifold with multidimensions. Therefore, cosmic string as well as superstring theories and hence M-theory which reproduce higher dimensional general relativity at low energy, argued that theories of unification tend to require extra spatial dimensions to be consistent with the physically viable models $[18,19,20,21,22,23$. The classical analogue of the effective String Theory is the low energy effective action containing squares and higher powers of curvature terms. Also, similar higher derivative gravitational terms appear in the renormalization of quantum field theory in curved space background. Further, it is shown that some features of higher dimensional black holes differ significantly from four dimensional black holes as higher dimensions allow for a much richer landscape of black hole solutions that do not have 4-dimensional counterparts 24. It draws more interest due to (1) a conceivable possibility of the production of higher dimensional black holes in future colliders in the scenario of large extra dimensions and TeV-scale gravity [25, 26], and (2) The AdS/CFT correspondence which relates the possibility of a $D$-dimensional black hole with those of a quantum field theory in $(D-1)$-dimensions $[27$.

In fact, the study of higher dimensional black holes have gained momentum in the first decade of this millennium. As in the present paper we are considering gravastar as an alternative to black holes so it is reasonable to adopt higher dimensional gravastar due to importance of higher dimensional black holes. Therefore, we present our study of higher dimensional gravastars proposed as an alternative to higher dimensional Schwarzschild-Tangherlini black holes 28 . We develop mathematical framework for these gravastars and obtain solutions for its three separable regions; the interior, the shell 
and the exterior. We then study proper length and energy, entropy and junction conditions in detail. The results and discussions have been presented in every section under various headings and subheadings. At the end, we conclude our findings.

\section{Interior space-time}

Since we are exploring for higher dimensional gravastar, we have assumed a $D$-dimensional spacetime with the structure $R^{1} X S^{1} X S^{d}(d=D-2)$, where $S^{1}$ is the range of the radial coordinate $r$ and $R^{1}$ is the time axis. For this purpose, let us consider a static spherically symmetric metric in $D=d+2$ dimension as

$$
d s^{2}=-e^{\nu} d t^{2}+e^{\lambda} d r^{2}+r^{2} d \Omega_{d}^{2}
$$

The notation, $d \Omega_{d}^{2}$ is a linear element on a $d$-dimensional unit sphere, parametrized by the angles $\phi_{1}, \phi_{2}, \ldots \ldots, \phi_{d}$ :

$$
d \Omega_{d}^{2}=d \phi_{d}^{2}+\sin _{2} \phi_{d}\left[d \phi_{d-1}^{2}+\sin _{2} \phi_{d-1}\left\{d \phi_{d-2}^{2}+\ldots \ldots \ldots+\sin _{2} \phi_{3}\left(d \phi_{2}^{2}+\right.\right.\right.
$$
$\left.\left.\left.\sin _{2} \phi_{2} d \phi_{1}^{2}\right) \ldots \ldots.\right\}\right]$.

The Hilbert action coupled to matter is given by

$$
I=\int d^{D} x \sqrt{-g}\left(\frac{R_{D}}{16 \pi G_{D}}+L_{m}\right),
$$

where $R_{D}$ is the curvature scalar in $D$-dimensional spacetime, $G_{D}$ denotes the $D$-dimensional Newton constant and $L_{m}$ is the Lagrangian for matter distribution. We obtain the following Einstein equation by varying the above action with respect to the metric as

$$
G_{a b}^{D}=-8 \pi G_{D} T_{a b},
$$

where $G_{a b}^{D}$ denotes the Einstein's tensor in $D$-dimensional spacetime.

The interior of the star is assumed to be perfect fluid type and can be given by

$$
T_{i j}=(\rho+p) u_{i} u_{j}+p g_{i j},
$$

where, $\rho$ represents the energy density, $p$ is the isotropic pressure, and $u^{i}$ is the $D$-velocity of the fluid. The Einstein field equations for the metric (11), together with the energy-momentum tensor given in Eq. (2), yield

$$
\begin{gathered}
-e^{-\lambda}\left[\frac{d(d-1)}{2 r^{2}}-\frac{d \lambda^{\prime}}{2 r}\right]+\frac{d(d-1)}{2 r^{2}}=8 \pi G_{D} \rho, \\
e^{-\lambda}\left[\frac{d(d-1)}{2 r^{2}}+\frac{d \nu^{\prime}}{2 r}\right]-\frac{d(d-1)}{2 r^{2}}=8 \pi G_{D} p
\end{gathered}
$$




$$
\begin{gathered}
\frac{e^{-\lambda}}{2}\left[\nu^{\prime \prime}-\frac{\lambda^{\prime} \nu^{\prime}}{2}+\frac{\nu^{\prime 2}}{2}-\frac{(d-1)\left(\lambda^{\prime}-\nu^{\prime}\right)}{r}+\frac{(d-1)(d-2)}{r^{2}}\right] \\
-\frac{(d-1)(d-2)}{2 r^{2}}=8 \pi G_{D} p,
\end{gathered}
$$

where a ' ' denotes differentiation with respect to the radial parameter $r$. Here we have assumed $c=1$ in geometrical unit. Conservation equation in $D$-dimensions implies

$$
\frac{1}{2}(\rho+p) \nu^{\prime}+p^{\prime}=0 .
$$

Following Mazur-Mottola [12, we assume the Equation of State (EOS) for the interior region in the form

$$
p=-\rho .
$$

Using this EOS, one gets from Eq. (8)

$$
\rho=\text { constant }=\rho_{c}, \quad(\text { say }) .
$$

We write this constant as, $\rho_{c}=d(d+1) \Lambda / 16 \pi G_{D}$, where $2 \Lambda / d(d+1)$ is the $D$-dimensional cosmological constant. This means that in the interior we are essentially considering the Cosmological Constant i.e. vacuum energy density of Einstein [29, 30].

Therefore, pressure may be expressed as follows

$$
p=-\rho_{c} .
$$

Using Eq. (9) one gets the solutions of $\lambda$ from the field Eq. (5) as given below

$$
e^{-\lambda}=1-\frac{16 \pi G_{D} \rho_{c}}{d(d+1)} r^{2}+E r^{1-d}
$$

where $E$ is an integration constant. Since $d>2$ and the solution is regular at $r=0$, so we demand $E=0$.

Using Eq. (9) one may obtain from Eqs. (5) and (6), the following relation

$$
\ln C=\lambda+\nu,
$$

where $\ln C$ is an integration constant. Thus we have the following interior solutions

$$
C e^{-\lambda}=e^{\nu}=C\left(1-\Lambda r^{2}\right) .
$$

We then calculate the active gravitational mass $M(r)$ in higher dimensions, which is found to be

$$
M(r)=\int_{0}^{r_{1}=R}\left[\frac{2 \pi^{\frac{d+1}{2}}}{\Gamma\left(\frac{d+1}{2}\right)}\right] r^{d} \rho d r=\left[\frac{2 \pi^{\frac{d+1}{2}}}{(d+1) \Gamma\left(\frac{d+1}{2}\right)}\right] \rho_{c} R^{d+1} .
$$

This is the usual gravitating mass for a $d$-dimensional sphere of radius $R$ and energy density $\rho_{c}$. The space-time metric thus obtained turns out to be free from any central singularity. 


\section{Exterior space-time}

The exterior region defined as $(p=\rho=0)$ in higher dimensions is nothing but a generalization of Schwarzschild solution, which as obtained by Tangherlini 28] reads as

$$
d s^{2}=-\left(1-\frac{\mu}{r^{d-1}}\right) d t^{2}+\left(1-\frac{\mu}{r^{d-1}}\right)^{-1} d r^{2}+d \Omega_{d}^{2} .
$$

Here $\mu=16 \pi G_{D} M / \Omega_{d}$ is the constant of integration with $M$, the mass of the black hole and $\Omega_{d}$, the area of a unit $d$-sphere as $\Omega_{d}=2 \pi^{\left(\frac{d+1}{2}\right)} / \Gamma\left(\frac{d+1}{2}\right)$.

\section{Shell}

It is assumed that thin shell contains ultra-relativistic fluid of soft quanta which obeys the EOS

$$
p=\rho .
$$

This represents stiff fluid model of Zel'dovich type in connection to cold baryonic universe 30.

It is difficult to obtain a general solution of the field equations in the non-vacuum region, i.e. within the shell. We try to find an analytic solution within the thin shell limit, $0<e^{-\lambda} \equiv h<<1$. As an advantage of it, we may set $h$ to be zero to the leading order. Under this approximation, the field Eqs. (57) - (7), with the above EOS, may be recast in the following form

$$
\begin{gathered}
\frac{h^{\prime}}{2 r}=\frac{(d-1)}{r^{2}}, \\
\frac{\nu^{\prime} h^{\prime}}{4}+\frac{(d-1) h^{\prime}}{2 r}=-\frac{(d-1)}{r^{2}} .
\end{gathered}
$$

Integration of Eq. (18) immediately yields

$$
h=E+2(d-1) \ln r,
$$

where $E$ is an integration constant. The range of $r$ lies within the thickness of the shell $\left[r_{1}=R, r_{2}=R+\epsilon\right]$. We, under the condition $\epsilon<<1$, get $E<<1$ as $h<<1$.

The other metric coefficient, $\nu$, can be found as

$$
e^{\nu}=\left(\frac{r}{r_{0}}\right)^{-2 d}
$$

where $r_{0}$ is an integration constant.

Also, from the conservation equation and using the same EOS as above, one may obtain

$$
p=\rho=\rho_{0} e^{-\nu}=\rho_{0}\left(\frac{r}{r_{0}}\right)^{2 d},
$$

$\rho_{0}$ being an integration constant. As $\rho \propto r^{2 d}$, so the ultra relativistic matter in the shell $\left(r_{1} \leq r<r_{2}\right)$ is more dense at the outer boundary $\left(r_{2}<r\right)$ than in the inner boundary $\left(0 \leq r<r_{1}\right)$. 


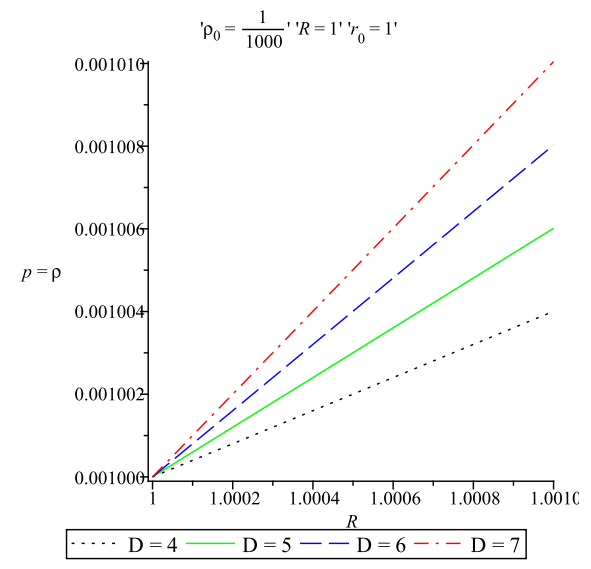

Fig. 1 The variation of pressure and density of the ultra relativistic matter in the shell against $r$ for different dimensions

\section{Proper length and Energy}

We consider matter shell is situated at the surface $r=R$, describing the phase boundary of region I. The thickness of the shell $(\epsilon<<1)$ is assumed to be very small. Thus the region III joins at the surface $r=R+\epsilon$.

Now, we calculate the proper thickness between two interfaces i.e. of the shell as

$$
\ell=\int_{R}^{R+\epsilon} \sqrt{e^{\lambda}} d r=\int_{R}^{R+\epsilon} \frac{d r}{[E+2(d-1) \ln r]^{\frac{1}{2}}} .
$$

By solving the above equation, one gets

$$
\ell=\left[a \frac{\sqrt{\pi} \operatorname{erf}\left[\sqrt{-a} \sqrt{\left(E+\frac{1}{a} \ln r\right)}\right]}{e^{a E} \sqrt{-a}}\right]_{R}^{R+\epsilon},
$$

where $a=\frac{1}{2(d-1)}$.

It will be interesting to calculate the energy $\widetilde{E}$ within the shell, which we find out as

$$
\begin{array}{r}
\widetilde{E}=\int_{R}^{R+\epsilon}\left[\frac{2 \pi}{\Gamma\left(\frac{d+1}{2}\right)}\right] r^{d} \rho d r=\int_{R}^{R+\epsilon}\left[\frac{2 \pi}{\Gamma\left(\frac{d+1}{2}\right)}\right] r^{d} \rho_{0}\left(\frac{r}{r_{0}}\right)^{2 d} d r \\
=\left[\frac{2 \pi \frac{d+1}{2}}{\Gamma\left(\frac{d+1}{2}\right)}\right]\left[\frac{\rho_{0}}{(3 d+1) r_{0}^{2 d}}\right]\left[(R+\epsilon)^{3 d+1}-R^{3 d+1}\right] .
\end{array}
$$

However, one may write the energy $\widetilde{E}$ within the shell up to first order in $\epsilon$ as

$$
\widetilde{E} \approx\left[\frac{2 \pi^{\frac{d+1}{2}}}{\Gamma\left(\frac{d+1}{2}\right)}\right] \rho_{0}\left(\frac{R}{r_{0}}\right)^{2 d} R \epsilon .
$$




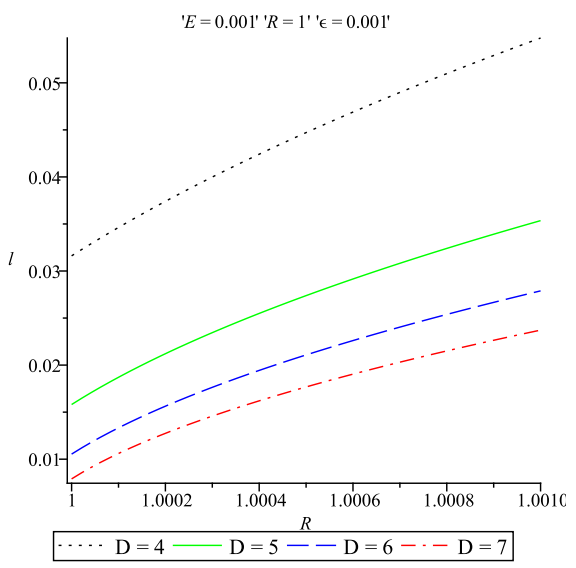

Fig. 2 The variation of proper length within the shell against $r$ for different dimensions

We observe that the energy within the shell is not only proportional to $\epsilon$ in first order of thickness but also depends on dimension $d$ of the spacetime.

\section{Entropy}

We calculate the entropy following Mazur and Mottola prescription [12] as

$$
S=\int_{R}^{R+\epsilon}\left[\frac{2 \pi^{\frac{d+1}{2}}}{\Gamma\left(\frac{d+1}{2}\right)}\right] r^{d} s(r) \sqrt{e^{\lambda}} d r .
$$

Here, $s(r)$ stands for the entropy density of the local temperature $T(r)$, which may be written as

$$
s(r)=\frac{\alpha^{2} k_{B}^{2} T(r)}{4 \pi \hbar^{2}}=\alpha\left(\frac{k_{B}}{\hbar}\right) \sqrt{\frac{p}{2 \pi}}
$$

where $\alpha^{2}$ is a dimensionless constant.

Thus the entropy of the fluid within the shell could be found as

$$
S=\int_{R}^{R+\epsilon}\left[\frac{2 \pi^{\frac{d+1}{2}}}{\Gamma\left(\frac{d+1}{2}\right)}\right] \sqrt{\frac{\alpha^{2} \rho_{0}}{2 \pi r_{0}^{2 d}}}\left(\frac{k_{B}}{\hbar}\right) \frac{r^{2 d} d r}{[E+2(d-1) \ln r]^{\frac{1}{2}}} .
$$

Solving the above equation, one gets

$$
S=\left[\left(\frac{2 \pi^{\frac{d+1}{2}}}{\Gamma\left(\frac{d+1}{2}\right)}\right) \sqrt{\frac{\alpha^{2} \rho_{0}}{2 \pi r_{0}^{2 d}}}\left(\frac{k_{B}}{\hbar}\right) \frac{1}{2(d-1)} \frac{\sqrt{\pi} \operatorname{erf}[\sqrt{-b} \sqrt{(E+2(d-1) \ln r)}]}{e^{b E} \sqrt{-b}}\right]_{R}^{R+\epsilon},
$$

where $b=\frac{2 d+1}{2(d-1)}$. 


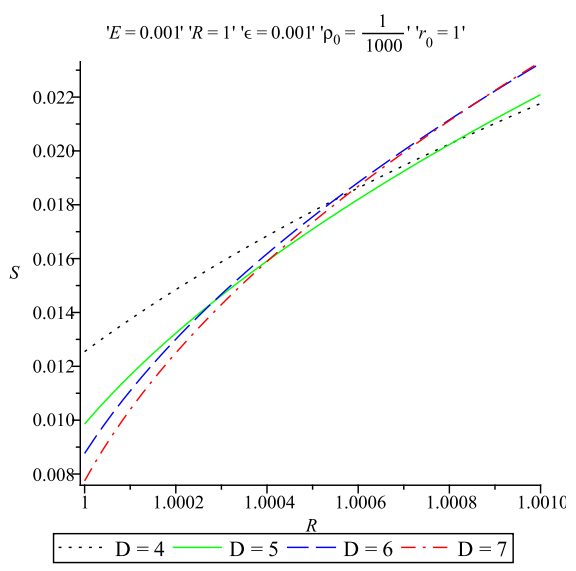

Fig. 3 The variation of Entropy within the shell against $r$ for different dimensions

\section{Junction Condition}

The gravastar configuration contains three regions in which interior region I is connected with exterior region II at the junction interface i.e. at the shell. This makes a geodecically complete manifold with a matter shell at the surface $r=R$. Thus a single manifold characterizes the gravastar configuration. According to fundamental junction condition there has to be a smooth matching between the regions I and III of the gravastar. However, though the metric coefficients are continuous at the junction surface $(S)$ their derivatives may not be continuous there. Thus affine connections may be discontinuous at the boundary surface, in other words, the second fundamental forms $31,32,33,34,35,36,37$.

$$
K_{i j}^{ \pm}=-n_{\nu}^{ \pm}\left[\frac{\partial^{2} x^{\nu}}{\partial \xi^{i} \partial \xi^{j}}+\Gamma_{\alpha \beta}^{\nu} \frac{\partial x^{\alpha}}{\partial \xi^{i}} \frac{\partial x^{\beta}}{\partial \xi^{j}}\right]_{\left.\right|_{S}},
$$

where, $n_{\nu}^{ \pm}$are the unit normals to $S$ and can be written as

$$
n_{\nu}^{ \pm}= \pm\left|g^{\alpha \beta} \frac{\partial f}{\partial x^{\alpha}} \frac{\partial f}{\partial x^{\beta}}\right|^{-\frac{1}{2}} \frac{\partial f}{\partial x^{\nu}} \quad \text { with } \quad n^{\mu} n_{\mu}=1
$$

which are associated with the two sides of the shell are discontinuous.

In Eq. (29), $\xi^{i}$ are the intrinsic coordinates on the shell and $f\left(x^{\alpha}\left(\xi^{i}\right)\right)=0$ is the parametric equation of the shell $S$. Here, - and + mention interior and exterior regions.

These discontinuity of the second fundamental forms,

$$
\kappa_{i j}=K_{i j}^{+}-K_{i j}^{-}
$$

produce intrinsic stress energy tensor within the shell. Using Lanczos equations $38,39,40,31,41,42$, one can write the surface intrinsic energy momentum tensors, $S_{i}^{j}=\operatorname{diag}(-\sigma,-v,-v, \ldots . .,-v)$ where 


$$
\sigma=-\frac{1}{8 \pi G_{D}} \kappa_{\tau}^{\tau}
$$

is the surface energy density and

$$
-v=\frac{1}{8 \pi G_{D}} \kappa_{\phi_{A}}^{\phi_{A}}
$$

is the surface tension.

For our gravastar configuration, we calculate

$$
\begin{gathered}
\sigma=-\frac{d}{8 \pi G_{D} R}\left[\sqrt{1-\frac{\mu}{R^{d-1}}}-\sqrt{1-\Lambda R^{2}}\right], \\
v=-\frac{1}{8 \pi G_{D}}\left[\frac{\frac{\mu(d-1)}{2 R^{d-1}}+(d-1)\left(1-\frac{\mu}{R^{d-1}}\right)}{\sqrt{1-\frac{\mu}{R^{d-1}}}}-\frac{-\Lambda R^{2}+(d-1)\left(1-\Lambda R^{2}\right)}{\sqrt{1-\Lambda R^{2}}}\right] .
\end{gathered}
$$

We see that the energy density as well as surface tension of the junction shell are negative. This means we have a thin shell of matter content with negative energy density. It is to be noted that the discontinuity of the affine connections at the region II i.e. in the shell provides the above matter confined within the shell. Such a stress-energy tensor is not ruled out from the consideration of Casimir effect between compact objects at arbitrary separations 43 . The above negative surface tension also indicates that there is a surface pressure as opposed to surface tension. Thus, in principle, the shell of our gravastar configuration consists of a combination of two types of matter distributions, namely, the ultra-relativistic fluid obeying $p=\rho$ and matter components due to discontinuity of second fundamental form of the junction interface, that are given in Eqs. (36) and (37). We demand that these two fluids are non-interacting and characterize the shell of the gravastar i.e. non-vacuum region II.

\section{Concluding remarks}

In the present work we generalize the concept of gravastar, a gravitational vacuum star, in the spacetime of 4-dimensional to Ddimensional Einstein gravity of the Schwarzschild-Tangherlini category black hole. To do so, firstly, we have considered three different regions with different EOS such as [I] $0 \leq r<r_{1}, \rho=-p$ (Interior), [II] $r_{1} \leq r<r_{2}, \rho=p$ (Shell) and [III] $r_{2}<r$, $\rho=p=0$ (Exterior). Secondly, the conjecture of electromagnetic mass (EMM) has been invoked due to the presence of charge. Originally Lorentz 14 proposed model for extended electron and conjectured that "there is no other, no 'true' or 'material' mass," and thus provides only 'electromagnetic masses of the electron'. Wheeler [15] and Wilczek [17 also argued that electron has a "mass without mass". Feynman, Leighton and Sands [16] termed this type of models as "electromagnetic mass models". Following the idea of EMM, where all the physical parameters, including the gravitational mass, 
are arising from the electromagnetic field alone, have been extensively studied by several investigators [44,45, 46, 47, 48, 49,50, 51,52, under the general relativistic framework where spacetime geometry is assumed to be associated with the presence of charged particle obeying Maxwell's equations of electromagnetic theory.

However, in connection to the interior configuration I of EMM we would like to record that most of the above investigators exploit an EOS with a repulsive pressure of the form $p=-\rho$ which is a very common feature in the context of the present accelerating Universe and have been argued to be connected with $\Lambda$-dark energy [53,54,55,56]. The EOS of this type implies that the matter distribution under consideration is in tension and hence the matter is known in the literature as a 'false vacuum' or 'degenerate vacuum' or ' $\rho$-vacuum' $[57,58,59,60$. This EOS was first discussed by Gliner 61. in his study of the algebraic properties of the energy-momentum tensor of ordinary matter through the metric tensors. Later on it was revealed that the gravitational effect of the zero-point energies of particles and electromagnetic fields are real and measurable, as in the Casimir Effect 62.

Whereas in connection to the shell configuration II it is to note that the stiff fluid model, which refers to a Zel'dovich universe, have been employed by several authors for various situations such as cold baryonic universe [30, early hadron era 63], scalar field fluid 64] and LRS Bianchi-I cosmological models 65 . There are also recent applications and claims for stiff fluid EOS in the various astrophysical systems like neutron star RX J1856-3754 [66], hyperon stars [67] and structure formation 68.

As a final remark we would like to add here that our sole aim in the present work was to find a classical analogue of the higher dimensional gravastar as an alternative to black holes and it seems that we are quite successful in our attempt.

\section{Acknowledgments}

FR, SC, SR and AAU wish to thank the authorities of the Inter-University Centre for Astronomy and Astrophysics, Pune, India for providing the Visiting Associateship under which a part of this work was carried out. FR is also thankful to UGC for providing financial support. We all are grateful to the referee for helpful suggestions which made us to upgrade the manuscript in a substantial manner.

\section{References}

1. Rahaman F. et al.: Phys. Lett. B 707319 (2012)

2. Rahaman F. et al.: Phys. Lett. B $\mathbf{7 1 7} 1(2012)$

3. Usmani A.A. et al.: Phys. Lett. B 701388 (2011)

4. Dymnikova I.G.: Gen. Relativ. Gravit. 24235 (1992)

5. Dymnikova I.: Int. J. Mod. Phys. D 5529 (1996)

6. Dymnikova I.G.: Phys. Lett. B 47233 (2000)

7. Dymnikova I.: Class. Quantum Gravit. 19725 (2002) 
8. Dymnikova I., Galaktionov E.: Phys. Lett. B 645358 (2007)

9. Dymnikova I., Korpusik M.: Phys. Lett. B 68512 (2010)

10. Dymnikova I., Galaktionov E.: Centr. Eur. J. Phys. 9644 (2011)

11. Dymnikova I., Fil'chenkov M.: AHEP 2013, Article ID 746894 (2013)

12. Mazur P., Mottola E.: arXiv:gr-qc/0109035, Report number: LA-UR-01-5067 (2001)

13. Mazur P., Mottola E.: Proc. Natl. Acad. Sci. USA 1019545 (2004)

14. Lorentz H.A.: Proc. Acad. Sci., Amsterdam 6 (1904) (Reprinted in The Principle of Relativity, Dover, INC., p. 24, 1952)

15. Wheeler J.A.: Geometrodynamics, Academic, New York, p. 25 (1962)

16. Feynman R.P., Leighton R.R., Sands M.: The Feynman Lectures on Physics, Addison-Wesley, Palo Alto, Vol. II, Chap. 28 (1964)

17. Wilczek F.: Phys. Today 5211 (1999)

18. Schwarz J.H.: Superstings, World Scientific, Singapore (1985)

19. Weinberg S.: Strings and Superstrings, World Scientific, Singapore (1986)

20. Duff M.J., Liu J.T., Minasian R.: Nucl. Phys. B 452261 (1995)

21. Polchinski J.: String Theory (Cambridge, 1998)

22. Hellerman S., Swanson I.: J. High Energy Phys. 0709096 (2007)

23. Aharony O., Silverstein E.: Phys. Rev. D 75046003 (2007)

24. Emparan R., Reall H.S.: Liv. Rev. Relativ. 116 (2008)

25. Cavaglia M.: Int. J. Mod. Phys. A, 181843 (2003)

26. Kanti P.: Int. J. Mod. Phys. A 194899 (2004)

27. Aharony O. et al.: Phys. Rept. 323183 (2000)

28. Tangherlini F.R.: Nuo. Cim. 27636 (1963)

29. Einstein A.: Sitz. Preuss. Akad. Wiss. 349 (Reprinted in The Principle of Relativity, Dover, INC., N.Y., 1952, p 190-198) (1919)

30. Zel'dovich Y.B.: Mon. Not. R. Astron. Soc. 1601 (1972)

31. Israel W.: Nuo. Cim. B 441 (1966); erratum - ibid. 48463 (1967)

32. Rahaman F. et al.: Gen. Relativ. Gravit. 381687 (2006)

33. Rahaman F. et al.: Acta Phys. Polon. B 401575 (2009)

34. Usmani A.A. et al.: Gen. Relativ. Gravit. 422901 (2010)

35. Rahaman F. et al.: Int. J. Theor. Phys. 492364 (2010)

36. Dias G.A.S., Lemos J.P.S.: arXiv:1008.3376 [gr-qc]

37. Rahaman F. et al.: Class. Quantum Gravit. 28155021 (2011)

38. Lanczos K.: ibid. 379518 (1924)

39. Sen N.: Ann. Phys. (Leipzig), 378365 (1924)

40. Darmois G.: Mémorial des Sciences Mathématiques, Fascicule XXV (GauthierVillars, Paris, 1927), Chap. 5

41. Perry G.P., Mann R.B.: Gen. Relativ. Gravit. 24305 (1992)

42. Musgrave P., Lake K.: Class. Quantum Grav. 131885 (1996)

43. Emig T. et al.: Phys. Rev. Lett. 99170403 (2007)

44. Florides P.S.: Proc. Camb. Phil. Soc. 58102 (1962)

45. Cooperstock F.I., de la Cruz V.: Gen. Relativ. Gravit. 9835 (1978)

46. Tiwari R.N., Rao J.R., Kanakamedala R.R.: Phys. Rev. D 30489 (1984)

47. Gautreau R.: Phys. Rev. D 311860 (1985)

48. Grøn Ø.: Phys. Rev. D 312129 (1985)

49. Ponce de Leon J.: J. Math. Phys. 28410 (1987)

50. Ray S., Das B.: Mon. Not. Roy. Astron. Soc. 3491331 (2004)

51. Ray S.: Int. J. Mod. Phys. D 15917 (2006)

52. Ray S. et al.: Ind. J. Phys. 821191 (2008)

53. Perlmutter S. et al.: Nat. 517565 (1998)

54. Riess A.G. et al.: Astron. J. 1161009 (1998)

55. Ray S., Mukhopadhyay U., Meng X.-H.: Gravit. Cosmol. 13142 (2007)

56. Usmani et al.: Mon. Not. R. Astron. Soc. 386 L92 (2008)

57. Davies P.C.W.: Phys. Rev. D 30737 (1984)

58. Blome J.J., Priester W.: Naturwiss. 71528 (1984)

59. Hogan C.: Nat. 310365 (1984)

60. Kaiser N., Stebbins A.: Nat. 310391 (1984)

61. Gliner E.B: Sov. Phys. JETP 22378 (1966)

62. Casimir H.B.G.: Proc. K. Ned. Acad. Wet. 51635 (1948) 
63. Carr B.J.: Astrophys. J. 2011 (1975)

64. Madsenet M.S. et al.: Phys. Rev. D 461399 (1992)

65. Chakrabarty I., Pradhan A.: Gravit. Cosmol. 755 (2001)

66. Braje T.M., Romani R.W.: Astrophys. J. 5801043 (2002)

67. Linares L.P., Malheiro M., Ray S.: Int. J. Mod. Phys. D 131355 (2004)

68. Buchert T.: Gen. Relativ. Gravit. 331381 (2001) 